\title{
Percutaneous aortic balloon valvuloplasty under echocardiographic guidance solely
}

\author{
Yongquan Xie, Shouzheng Wang, Guangzhi Zhao, Muzi Li, Fengwen Zhang, Wenbin Ouyang, Xiangbin Pan \\ Department of Cardiovascular Surgery, National Center for Cardiovascular Disease, China \& Fuwai Hospital, Chinese Academy of Medical Sciences \\ \& Peking Union Medical College, Beijing 100037, China \\ Contributions: (I) Conception and design: Y Xie, X Pan; (II) Administrative support: Y Xie, S Wang; (III) Provision of study materials or patients: Y \\ Xie, G Zhao, M Li, W Ouyang, X Pan; (IV) Collection and assembly of data: F Zhang; (V) Data analysis and interpretation: Y Xie, F Zhang; (VI) \\ Manuscript writing: All authors; (VII) Final approval of manuscript: All authors. \\ Correspondence to: Dr. Xiangbin Pan. National Center for Cardiovascular Disease, China \& Fuwai Hospital, Chinese Academy of Medical Sciences \& \\ Peking Union Medical College, 167 Beilishi Rd, Xicheng District, Beijing 100037, China. Email: panxiangbin@fuwaihospital.org.
}

\begin{abstract}
Background: Percutaneous balloon aortic valvuloplasty (PBAV) is an alternative to surgical valvulotomy for the treatment of congenital aortic stenosis (AS). This article aims to summarize our preliminary experience on feasibility and safety of PBAV under only echocardiographic guidance in patients with congenital AS.

Methods: Clinical data from 20 consecutive patients with aortic valve stenosis who underwent PBAV under only echocardiographic guidance at Fuwai Hospital from January 2016 to January 2019 were analyzed retrospectively. Median age of patients was $18.38 \pm 15.88$ years and $65 \%$ of the patients were male. Aortic annulus diameter was $18.40 \pm 3.25 \mathrm{~mm}$ and balloon diameter was $17.38 \pm 3.89 \mathrm{~mm}$, with B/A ratio of $0.93 \pm 0.06$. Results: All the patients successfully underwent PBAV. The peak transaortic gradient (TG) significantly decreased from $81.59 \pm 24.91$ (range, $58-112$ ) $\mathrm{mmHg}$ preoperatively to $36.32 \pm 12.83$ (range, 16-51) $\mathrm{mmHg}$ $(\mathrm{P}=0.000)$ immediately post operation, without significant difference in aortic regurgitation (AR). At mean $24.31 \pm 17.35$ months follow-up, peak TG was 37.06 \pm 13.52 (range, 21-58) $\mathrm{mmHg}$ which was not significantly different from the immediate postoperative value $(\mathrm{P}=0.65)$.

Conclusions: In this retrospective, single center study, systematic use of Doppler echocardiography as only guidance modality for PBAV was feasible and associated with a high success rate and a very low complication rate.
\end{abstract}

Keywords: Aortic stenosis (AS); echocardiography; angiography; percutaneous intervention; aortic valvuloplasty

Submitted Aug 13, 2019. Accepted for publication Dec 10, 2019.

doi: $10.21037 /$ jtd.2020.01.16

View this article at: http://dx.doi.org/10.21037/jtd.2020.01.16

\section{Introduction}

Percutaneous balloon aortic valvuloplasty (PBAV) is an alternative to surgical valvulotomy for the treatment of congenital aortic stenosis (AS) (1). Although PBAV is traditionally conducted under angiographic guidance, in recent years, Doppler echocardiography has been increasingly used in the catheterization laboratory to guide congenital heart disease interventions, however, few cases of successful BAV guided solely by transesophageal echocardiography (TEE) have been reported (2-4). We have performed successfully non-fluoroscopy guided interventions for patients who are pregnant or suffer from aplastic anemia, allergy to iodine, or renal dysfunction, and developed progress for patients without contraindications for fluoroscopy (5-10). Here we report on a series of 20 consecutive patients who underwent PBAV guided solely by continued echocardiography at our institution.

\section{Methods}

\section{Patients}

From January 2016 to January 2019 a total of 20 consecutive 
patients with congenital AS underwent PBAV at our hospital. Median age was $18.38 \pm 15.88$ (range, 4-36) years and $65 \%$ of the patients were male. One thing to note is that a 27-year-old primigravida at the 16th week of gestation was treated by this procedure. The echocardiography showed $35 \%$ of patients with bicuspid malformation. The preoperative clinical data are summarized in Tables 1 and 2. Indications for PBAV included severe AS with the presence of a peak Doppler gradient $>70 \mathrm{mmHg}$ irrespective of symptoms or $>50 \mathrm{mmHg}$ with clinical symptoms and/or an electrocardiographic (ECG) strain or a critical AS with heart failure. Contraindications included asymptomatic

Table 1 Perioperative and operative characteristics of patients

\begin{tabular}{lc}
\hline Variable & Value \\
\hline Number of patients & 20 \\
Age (years) & $18.38 \pm 15.88$ \\
Weight (kg) & $41.50 \pm 19.62$ \\
Male sex & $13(65 \%)$ \\
Bicuspid aortic valve & $7(35 \%)$ \\
LVEF (\%) & $62.89 \pm 17.42$ \\
Aortic annulus diameter (mm) & $18.40 \pm 3.25$ \\
Balloon diameter (mm) & $17.38 \pm 3.89$ \\
Balloon/annulus ratio & $0.93 \pm 0.06$ \\
Intraoperative resuscitation & $1(5 \%)$ \\
TTE with local anesthesia & $18(90 \%)$ \\
TEE with general anesthesia & $2(10 \%)$ \\
In hospital complications & 0 \\
\hline
\end{tabular}

Data are presented as $n(\%)$ or mean \pm SD. LVEF, left ventricular ejection fraction; TTE, transthoracic echocardiography; TEE, transesophageal echocardiography. patients with AS whose catheterized transaortic peak gradient was $<40 \mathrm{mmHg}$, without ECG change or combined with moderate to severe regurgitation, warranting aortic replacement or repair. Mild AR was defined by the color jet ending proximal to the tip of the anterior mitral valve leaflet with a jet width $<25 \%$ of the left ventricular outflow tract diameter. Moderate AR was defined by the color jet ending distal to the mitral valve with a jet width $>25$ and $<65 \%$ of the left ventricular outflow tract diameter. Severe AR was defined by a color jet ending distal to the mitral valve with a jet width of $65 \%$ of the left ventricular outflow tract diameter. The study protocol was approved by the institutional Ethics Committee of Fuwai Hospital (No. 2018-8) and all patients, parents or legal guardians signed informed consent for the operation and clinical record review.

\section{Procedure}

Local 1\% lidocaine at the access site in the right groin was used routinely in patients undergoing transthoracic echocardiography (TTE) guided PBAV. TEE with general anesthesia and intubation was used as alternative for patients with poor echo view by TTE. Routine reassessment of aortic ring and transvalvular gradient was mandatory at the beginning of the procedure (Figure 1). The right jugular vein was punctured and a $6 \mathrm{~F}$ arterial sheath was introduced. A temporary pace wire was advanced to the right ventricle through the sheath and linked to a temporary pacemaker. The right femoral artery was punctured, an a $6 \mathrm{~F}$ pigtail catheter and guiding wire were delivered via aortic arch and ascending aorta to superior plane to aortic valve in suprasternal view. The guidewire was retained and the pigtail catheter was withdrawn; the length of pigtail catheter in vivo was marked as first working distance. A

Table 2 Comparison between preoperative and immediately postoperative values of clinical parameters

\begin{tabular}{lccc}
\hline Variable & Preop & Postop & P value \\
\hline Number of patients & 20 & 20 & - \\
Aortic regurgitation & $9(45 \%)$ & $12(60 \%)$ & 0.064 \\
Trivial & 7 & 9 & \\
Mild & 2 & 3 & 0.000 \\
Peak TG by catheter $(\mathrm{mmHg})$ & $74.25 \pm 31.41$ & $25.13 \pm 19.48$ & 0.000 \\
Peak TG by Echo $(\mathrm{mmHg})$ & $81.59 \pm 24.91$ & $36.32 \pm 12.83$ & \\
\hline
\end{tabular}

Data are presented as $n(\%)$ or mean \pm SD. TG, transaortic gradient. 


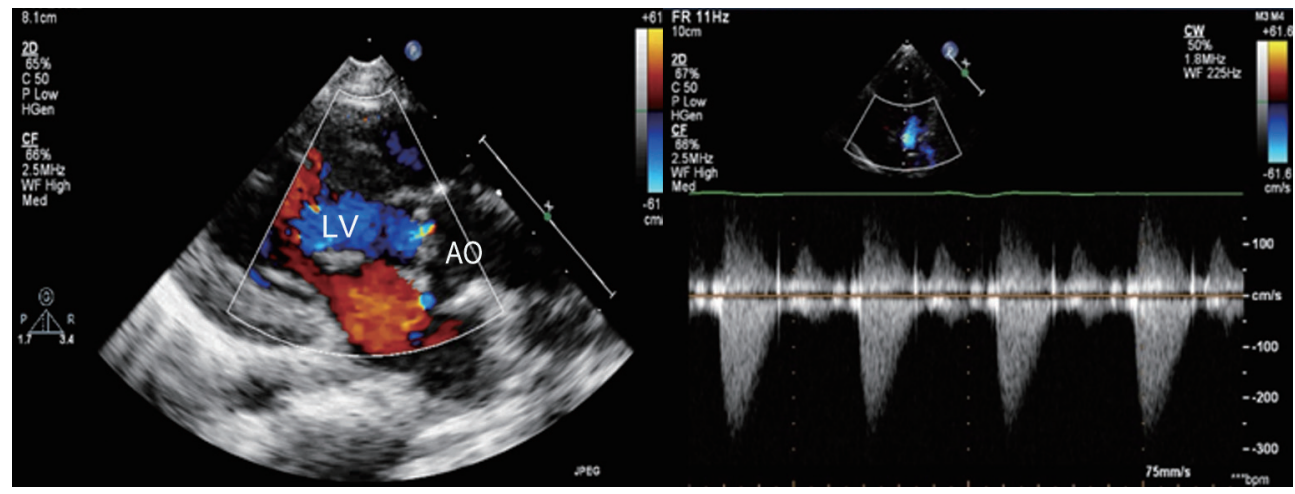

Figure 1 Preprocedural assessment of aortic valve stenosis degree.

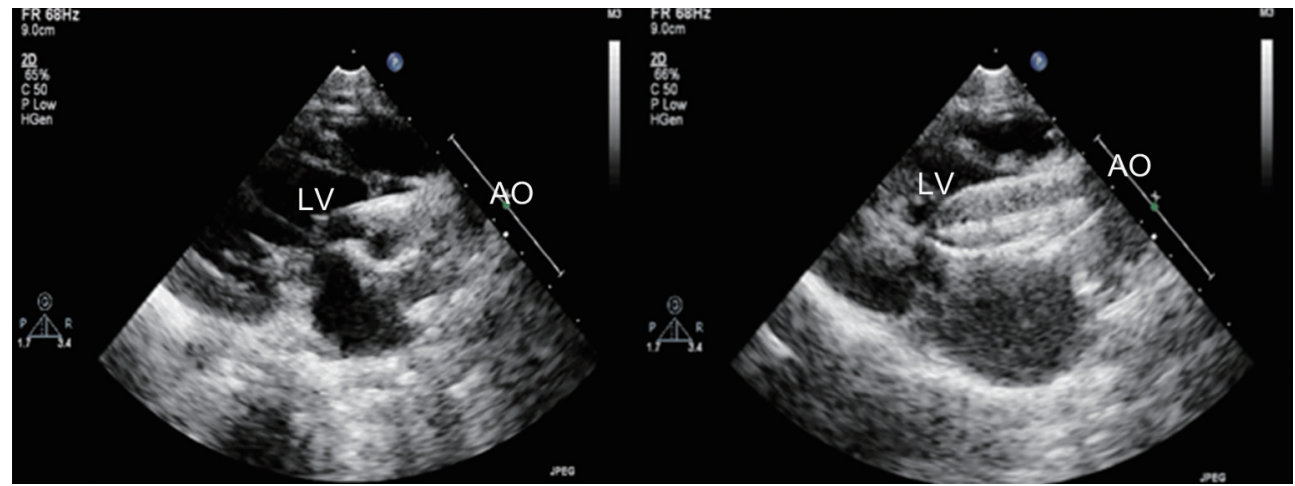

Figure 2 Image showing that the balloon catheter had passed through the stenotic aortic valve. The balloon is inflated across the aortic valve.

MPA2 catheter was inserted to a depth corresponding to the first working distance, and the aortic pressure was measured. Under guidance of echocardiography, the catheter and wire were advanced through the aortic orifice into the left ventricle, and the $\mathrm{LV}$ pressure was measured; transaortic gradient (TG) was determined by comparing left ventricular and aortic pressure. Evaluation of TG by catheter was unnecessary in patients with critical AS when there was hemodynamic deterioration such as reduction of heart rate and aortic pressure because of unenduring aortic orifice obstruction by catheter. The MPA2 catheter was withdrawn and the guidewire was left in the left ventricle; the MPA2 catheter length in vivo was marked as the second working distance. Balloon diameter did not exceed aortic ring diameter, starting with dilation of a smaller balloon and gradually increasing balloon diameter. The overall goal was to reduce the peak gradient to as low as possible without causing AR. The balloon was advanced using the guidewire until it reached the second working distance, and the balloon was slowly adjusted to ensure that aortic ring was located at the middle of the balloon. Using rapid pacing while holding the balloon and wire, the balloon was quickly inflated to dilate the stenotic aortic valve (Figure 2). The balloon was deflated after dilation and withdrawn into the ascending aorta; and aortic valve movement was evaluated by Doppler (Figure 3). A larger balloon was used for redilation if the results of the previous dilation were unsatisfactory. The procedure was terminated if: TG was $\leq 40 \mathrm{mmHg}$, gradient reduction was $\geq 50 \%$ compared to baseline, moderate to severe AR developed, or a B/ A ratio was 1.0 irrespective of the hemodynamic results. The balloon, guidewire and sheath were removed after a satisfactory dilation effect was achieved; and a vascular suture device was used for hemostasis of access site. 


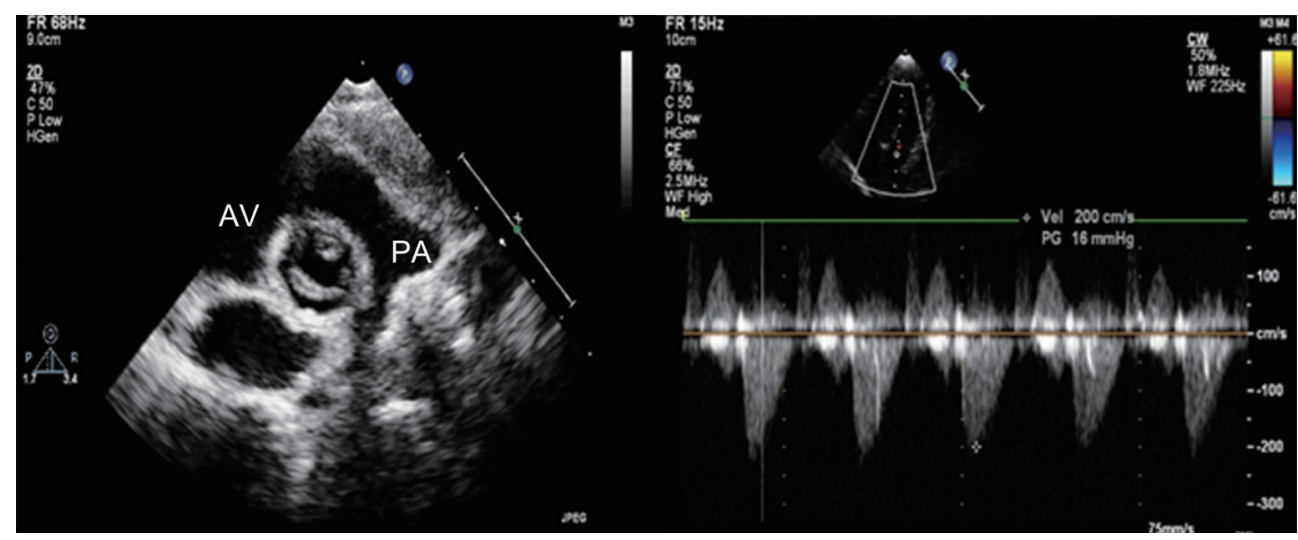

Figure 3 Immediate assessment of balloon aortic valvuloplasty results.

\section{Follow-up}

Follow-up was obtained for all patients. Clinical data were recorded at the latest follow-up and included the presence of clinical symptoms, and Doppler echocardiography peak and mean TG. Cardiac events were defined as cardiac death and aortic valve reintervention. Death was considered of cardiac origin unless proven otherwise.

\section{Statistical analysis}

Continuous variables are expressed as means \pm standard deviation and ranges and were compared using the pairedsamples $t$-test, while categorical variables are expressed as frequencies and percentages and were compared using the Chi-square test. SPSS 18.0 for Windows (SPSS Inc., Chicago) was used for statistical analysis. A P value of less than 0.05 was considered significant.

\section{Results}

\section{Preoperative and immediately postoperative results (Tables 1,2)}

All 20 patients successfully underwent PBAV. Two patients were converted to TEE guidance and general anesthesia due to poor quality of TTE image. Aortic annulus diameter was $18.40 \pm 3.25 \mathrm{~mm}$ and balloon diameter was $17.38 \pm 3.89 \mathrm{~mm}$. Varying degrees of pericardial effusion were observed preoperatively in 5 patients. Combined abnormalities included mild subaortic membrane (without indication of intervene) in 2 patients, simultaneous ductus artery occlusion and subaortic membrane in 1 patient, mild mitral stenosis in 1 patient, and mild mitral regurgitation in 3 patients. Before operation, 7 patients had trivial aortic valve regurgitation and 2 had mild AR. One patient was successfully resuscitated from severe hemodynamic deterioration right after deflation. The peak TG decreased significantly from $81.59 \pm 24.91$ (range, $58-112$ ) $\mathrm{mmHg}$ preoperatively to $36.32 \pm 12.83$ (range, $16-51$ ) $\mathrm{mmHg}$ $(\mathrm{P}=0.000)$ immediately post operation. Aortic regurgitation (AR) was trivial in 9 patients and mild in 3 patients immediately after dilation, without significant difference with preoperative rate $(\mathrm{P}=0.064)$. Only one patient remained mild mitral regurgitation and the other two were improved to trivial regurgitation. There were no other complications, such as vascular injury, cardiac tamponade or systematic embolism.

\section{Midterm outcomes}

There were no deaths during hospitalization or followup. At mean 24.31 17.35 (range, 6-42) months follow-up, peak TG was $37.06 \pm 13.52$ (range, $21-58$ ) $\mathrm{mmHg}$ without significant difference with value immediately post operation $(\mathrm{P}=0.65)$. All the patients showed good cardiac function without new onset $A R$, and $A R$ remained stable in those with pre-existing AR. Trival mitral regurgitation was found in two patients. Reintervention or surgery was unnecessary in any patient. That pregnant woman got a safe delivery and her baby was in normal status.

\section{Discussion}

In this retrospective single center study with a very limited 
number of patients and only midterm follow up duration, PBAV under echocardiographic guidance was feasible and safe.

Balloon aortic valvuloplasty (BAV) for the management of patients with severe congenital AS, was first reported in 1984 by Lababidi, as an "alternative treatment whenever surgical valve replacement proved impracticable" (1), even if in the era of transcatheter aortic valve replacement, PBAV still owns its practicability. The BAV technique has experienced minor changes since its introduction and use of angiographic guidance has prevailed; however, Doppler echocardiography has been used increasingly in cardiac catheterization laboratories to guide congenital heart disease interventions (2-4). Because interventional cardiac catheterizations are among the radiological procedures with the highest patient and physician X-ray radiation dose (10), many studies have demonstrated higher incidence of chromosomal damage, a marker of carcinogenesis, cancer and even patient cardiovascular damage (10-15). To meet the needs of avoiding detrimental radiation exposure of both doctors and patients, no contrast agent use, cost savings, and technique simplification with outcome improvement, we have developed a percutaneous nonfluoroscopy-guided procedure (PAN's procedure) for atrial septal defect, ventricular septal defect, patent ductus arteriosus, pulmonary stenosis and mitral stenosis, which is routinely performed using echocardiography as only guidance modality at our center (5-9). The latter earlier experiences helped us to successfully proceed to solely echocardiography guided PBAV.

PBAV under only echocardiographic guidance involves some technical considerations. General anesthesia and intubation should be considered in patients with severe cardiac dysfunction because balloon inflation may affect LVOT and remarkably reduce coronary blood supply, possibly leading to fatal systolic dysfunction or circulation crash. We perform all the procedures in a hybrid operation room with cardiac surgery team standby. If extracardiac resuscitation fails 15-20 seconds after balloon deflation, it must be converted to intracardiac resuscitation by median sternotomy.

The working length is a very important and meaningful conception. With the difference to angiographic guidance, the guidewire and catheter cannot be monitored precisely during advancement from puncture site to aortic arch in echo guided PBAV. Measurement of working length provides operator a reference length to advance guidewire and catheter, thus avoids vascular, valvular and ventricular complications (5-9).

Two technical key points of passage through a stenotic aortic orifice should be noted. Firstly, the strong high velocity blood stream crossing the aortic orifice renders passage of the guiding wire difficult. Thicker and harder wires such as superstiff or catheter may offer better directivity to cross through the aortic orifice. Secondly, to place the catheter as close as possible to the aortic orifice plane, the wire should be pushed and delivered during the cardiac systolic phase. Here echocardiography, especially combined 3D image, offers an advantage over angiography which does not allow to continuously judge the aortic orifice plane and to capture the opening and closing movement of the valve.

The development of postoperative AR remains the most important complication and risk factor for long term results (16-20). Patient's age between 1 and 5 years, prior valvulotomy, uni- and bi-commissural valve, aortic annulus $<16 \mathrm{~mm}$, and the presence of mild AR before PBAV are predictors of postoperative moderate to severe AR (15-17). However, the most important risk factor is an oversized balloon leading to a B/A ratio $>100 \%(16,17)$. Moreover, Christine first revealed that angiographic measurements significantly overestimated aortic annulus diameters (mean overestimation $>2 \mathrm{~mm}$ ) compared to echocardiographic measurements (4), which might underlie the significant incidence with angiographic guidance of post-dilation AR even in patients of $\mathrm{B} / \mathrm{A}$ ratio $<100 \%$. The latter limitation strongly favors use of aortic echocardiographic measurement of the aortic annulus to inform balloon size (4). The lack of a significant difference between preoperative and postoperative $\mathrm{AR}$ in this study might translate into better long-term outcomes with echocardiographic than angiographic guidance. We measure the aortic annulus by echocardiography only to choose balloon size at $80-90 \%$ of the diameter of the aortic annulus maintaining $\mathrm{B} / \mathrm{A}$ ratio at $<100 \%$.

The advantages provided by echocardiography guidance of simultaneously and continuously judging aortic orifice plane, confirming wire position and direction, and accurately monitoring valve leaflet movement, renders passage of wire and catheter easier and more accurate relative to angiographic guidance. The latter advantages translate into less damage to the valve leaflet. Moreover, even imperceptible aortic valve leaflet perforation by the guidewire can be detected by continuous echocardiographic guidance thereby avoiding further faulty balloon manipulation and dilation in a false aortic valve lumen, which also may lead to acute significant AR. Last but not 
least, the greater precision in determining aortic annulus plane by echocardiography aids in precisely localizing balloon to the right position, thereby avoiding valve damage by balloon sliding. All the latter advantages result in a lower incidence of post-dilation AR.

The present study has the limitations inherent to its retrospective single center design with a relatively small sample size. Further larger prospective and comparative studies with longer-term follow-up may reveal whether PBAV under sole echocardiographic guidance should become a new alternative access for congenital AS.

\section{Conclusions}

Percutaneous aortic balloon valvuloplasty solely guided by echocardiography is one of the Percutaneous and nonfluoroscopical procedures with unique advantages and challenges. In the present study, systematic use of Doppler echocardiography guidance solely for PBAV interventions was a feasible alternative access for treatment of congenital AS and associated with a high success rate, and a very low complication rate. Larger, appropriately powered, multicenter studies with longer-term follow-up are warranted to confirm these findings.

\section{Acknowledgments}

Funding: The National Key Research and Development Program of China (2016YFC1302004).

\section{Footnote}

Conflicts of Interest: The authors have no conflicts of interest to declare.

Ethical Statement: The authors are accountable for all aspects of the work in ensuring that questions related to the accuracy or integrity of any part of the work are appropriately investigated and resolved. The study protocol was approved by the institutional Ethics Committee of Fuwai Hospital (No. 2018-8) and all patients, parents or legal guardians signed informed consent for the operation and clinical record review.

Open Access Statement: This is an Open Access article distributed in accordance with the Creative Commons Attribution-NonCommercial-NoDerivs 4.0 International License (CC BY-NC-ND 4.0), which permits the non- commercial replication and distribution of the article with the strict proviso that no changes or edits are made and the original work is properly cited (including links to both the formal publication through the relevant DOI and the license). See: https://creativecommons.org/licenses/by-nc-nd/4.0/.

\section{References}

1. Lababidi Z, Wu JR, Walls JT. Percutaneous balloon aortic valvuloplasty: Results in 23 patients. Am J Cardiol 1984,53:194-7.

2. Weber HS, Mart CR, Kupferschmid MJ, et al. Transcarotid balloon valvuloplasty with continuous transesophageal echocardiographic guidance for neonatal critical aortic valve stenosis: an alternative to surgical palliation. Pediatr Cardiol 1998;19:212-7.

3. Patel S, Saini AP, Nair A, et al. Transcarotid balloon valvuloplasty in neonates and small infants with critical aortic valve stenosis utilizing continuous transesophageal echocardiographic guidance: A 22 year single center experience from the cath lab to the bedside. Catheter Cardiovasc Interv 2015;86:821-7.

4. Bourgault C, Rodés-Cabau J, Côté JM, et al. Usefulness of Doppler echocardiography guidance during balloon aortic valvuloplasty for the treatment of congenital aortic stenosis. Int J Cardiol 2008;128:30-7.

5. Pan X, Ouyang W, Li S, et al. Safety and efficacy of percutaneous patent ductus arteriosus closure solely under thoracic echocardiography guidance. Zhonghua Xin Xue Guan Bing Za Zhi 2015,43:31-3.

6. Liu Y, Guo GL, Ouyang WB, et al. Feasibility and effectiveness of percutaneous ventricular septal defect closure under solely guidance of echocardiography. Zhonghua Yi Xue Za Zhi 2017;97:1222-6.

7. Pan XB, Ouyang WB, Pang KJ, et al. Percutaneous Closure of Atrial Septal Defects Under Transthoracic Echocardiography Guidance Without Fluoroscopy or Intubation in Children. J Interv Cardiol 2015;28:390-5.

8. Wang SZ, Ouyang WB, Hu SS, et al. First-in-Human Percutaneous Balloon Pulmonary Valvuloplasty Under Echocardiographic Guidance Only. Congenit Heart Dis 2016;11:716-20.

9. Liu Y, Guo GL, Wen B, et al. Feasibility and effectiveness of percutaneous balloon mitral valvuloplasty under echocardiographic guidance only. Echocardiography. 2018;35:1507-11.

10. Bacher K, Bogaert E, Lapere R, et al. Patient specific dose and radiation risk estimation in pediatric cardiac 
catheterization. Circulation 2005;111:83-9.

11. Cohen S, Liu A, Gurvitz M, et al. Exposure to LowDose Ionizing Radiation from Cardiac Procedures and Malignancy Risk in Adults with Congenital Heart Disease. Circulation 2018;137:1334-45.

12. Baysson H, Rehel JL, Boudjemline Y, et al. Risk of cancer associated with cardiac catheterization procedures during childhood: a cohort study in France. BMC Public Health 2013;13:266.

13. Meinel FG, Nance JW Jr, Harris BS, et al. Radiation risks from cardiovascular imaging tests. Circulation 2014;130:442-5

14. Andreassi MG, Ait-Ali L, Botto N, et al. Cardiac catheterization and long-term chromosomal damage in children with congenital heart disease. Eur Heart J 2006;27:2703-8.

15. Modan B, Keinan L, Blumstein T, et al. Cancer following cardiac catheterization in childhood. Int J Epidemiol

Cite this article as: Xie Y, Wang S, Zhao G, Li M, Zhang F, Ouyang W, Pan X. Percutaneous aortic balloon valvuloplasty under echocardiographic guidance solely. J Thorac Dis 2020;12(3):477-483. doi: 10.21037/jtd.2020.01.16
2000;29:424-8.

16. McCrindle BW. Independent predictors of immediate results of percutaneous balloon aortic valvulotomy in children. Am J Cardiol 1996;77:286-93.

17. Sholler GF, Keane JF, Perry SB, et al. Balloon dilation of congenital aortic valve stenosis. Results and influence of technical and morphological features on outcome. Circulation 1988;78:351-60.

18. Moore P, Egito E, Mowrey H, et al. Midterm results of balloon dilation of congenital aortic stenosis: predictors of success. J Am Coll Cardiol 1996;27:1257-63.

19. Balmer C, Beghetti M, Fasnacht M, et al. Balloon aortic valvoplasty in paediatric patients: progressive aortic regurgitation is common. Heart 2004;90:77-81.

20. Reich O, Tax P, Marek J, et al. Long term results of percutaneous balloon valvuloplasty of congenital aortic stenosis: independent predictors of outcome. Heart 2004;90:70-6. 